\title{
SAÚDE INDÍGENA EM TEMPO DE COVID-19: O PROTAGONISMO DA ENFERMAGEM
}

Esron Soares Carvalho Rocha' ORCID: 0000-0002-1011-6053

Rizioléia Marina Pinheiro Pina' ORCID: 0000-0002-6114-4003

Marcelle Collyer da Silveira" ORCID:0000-0002-5623-9067

Edinilza Ribeiro dos Santos"'I ORCID: 0000-0002-3188-0114

Noeli Toledo das Neves' ORCID:0000-0001-5624-1813

'Universidade Federal do Amazonas. Manaus, Amazonas, Brasil.

"Universidade do Estado do Amazonas. Manaus, Amazonas, Brasil. Distrito Sanitário Especial Indígena. Manaus, Amazonas, Brasil.

"' Universidade do Estado do Amazonas. Manaus, Amazonas, Brasil.

Autor Correspondente: Esron Soares Carvalho Rocha E-mail: erocha@ufam.edu.br

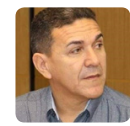

Como citar:

Rocha ESC, Pina RMP, Silveira MC, Santos ER, Neves NT. Saúde indígena em tempo de covid-19: o protagonismo da enfermagem. In: Teodósio SSS, Leandro SS (Orgs.). Enfermagem na atenção básica no contexto da COVID-19. 2.ed.rev. Brasília, DF : Editora ABEn, 2020. p. 73-80. (Série Enfermagem e Pandemias, 3). https://doi.org/10.51234/aben.20.e03.c11

\section{INTRODUÇÃO}

Ao atingir o Brasil em fevereiro de 2020, a pandemia de infecção humana causada pelo Novo Coronavírus (SARS-CoV-2) colocou o Estado do Amazonas entre os cinco estados da Federação com maior número de casos confirmados e de óbitos por Corona Vírus Diesease (COVID-19). Isso levou o sistema de saúde do estado ao colapso, no âmbito do SUS. Em relação a todos os estados, o AM teve, até 29/06/2020, a maior taxa de incidência acumulada de mortalidade por COVID-19 (67,4/100.000 habitantes). Cabe também destacar que a Região Norte ocupou, até a data cotada, a primeira posição na proporção de óbitos por COVID-19 em comparação às outras regiões do País, com 51 óbitos/100.000 habitantes (a média para o Brasil no mesmo período é de 27,7 óbitos/100.000 habitantes) ${ }^{(1,2)}$.

Indicadores sociais de abrangência nacional classificam a Região Norte como pertencente à classe "E" de vulnerabilidade social, por ser constituída por extensas áreas rurais, baixa densidade demográfica, com índice de desenvolvimento humano muito baixo, acesso precário à água tratada, esgoto e eletricidade, entre outros resultados negativos. Em relação às Regiões Sul e Sudeste do País, a Norte tem menor capacidade de resposta aos problemas de saúde, em termos de estrutura da Rede de Atenção à Saúde (RAS), e em particular aos casos graves de COVID-19(3). Isso contribuiu imensamente para a fase de aceleração descontrolada da transmissão e dos patamares alcançados pelos números de casos e óbitos confirmados por COVID-19 na população geral.

O Subsistema de Saúde Indígena, integrado ao Sistema Único de Saúde (SUS) e sob a gestão da Secretaria Especial de Saúde Indígena (SESAI), está organizado em 34 Distritos Sanitários Especiais IndígenasDSEls no território nacional. Destes, 19 estão localizados na Região Norte, sendo sete em território amazonense ${ }^{(4)}$ e um deles é o DSEI-Manaus. No âmbito estadual (AM) concentra-se cerca de 18\% da 
população indígena brasileira, residindo dentro ou fora de Terras Indígenas demarcadas(5). O DSEI-Manaus está organizado em 17 Polos-Base, abrangendo 19 municípios ${ }^{(4)}$.

A atenção primária à saúde (APS) nos DSEls segue o modelo da Estratégia Saúde da Família. Assim, as ações de saúde nos territórios indígenas são desenvolvidas por equipes multidisciplinares, sendo a presença de trabalhadores de enfermagem no território permanente e em tempo integral. Além do contato direto com a comunidade, esses profissionais fazem a interlocução com os demais membros da equipe de saúde e mantêm diálogo com unidades de referência para atendimento dos indígenas no âmbito do SUS. Neste cenário, os profissionais de enfermagem também desenvolvem habilidades de gestão do cuidado (planejamento das intervenções, gestão de pessoal), de assistência (organização, execução e avaliação das práticas de enfermagem) e de ensino (práticas de educação permanente, educação em saúde nos âmbitos das comunidades locais, Polos-Base e DSEls), levando em conta a diversidade étnica dos povos indígenas ${ }^{(6)}$.

No âmbito APS e no contexto da pandemia da COVID-19 entre os povos indígenas, os profissionais de enfermagem realizam orientaçõessobre a higienização das mãos, para adotarem a etiqueta da tosse e a higiene respiratória, uso de máscaras, distanciamento social e outras medidas de prevenção. Também orientam quanto à identificação precoce de sinais e sintomas da COVID-19 e encaminhamento aos serviços de saúde dos casos graves ${ }^{(7)}$.

A fim de implantar estratégias para uma atuação coordenada das equipes multidisciplinaresem todo o território do DSEl-Manaus, foi elaborado um Plano de Contingenciamento para Infecção Humana pelo Novo Coronavírus entre Povos Indígenas. Da elaboração e implantação desse plano de trabalho participaram enfermeiros da gestão, assistência e pesquisa da área de saúde indígena.

Esse cenário, marcado pelo surto pandêmico do Novo Coronavírus, a especificidade da saúde indígena e a interface das práticas de enfermagem, motivou o interesse em relatar acerca do protagonismo da enfermagem durante o processo de planejamento e intervenção nos territórios indígenas, para a prevenção da COVID-19 entre essas populações vulneráveis.

\section{OBJETIVO}

Relatar a experiência de profissionais e pesquisadores enfermeiros na sistematização de ações de promoção da saúde, cuidado integral e segurança de povos indígenas e trabalhadores no enfrentamento da COVID-19, no Distrito Sanitário Especial Indígena de Manaus.

\section{MÉTODO}

Trata-se de relato de experiência sobre a organização de práticas desempenhadaspela enfermagem no enfrentamento da pandemia causada pelo Novo Coronavírus entre povos indígenas do DSEI-Manaus. A ordem dos elementos essenciais que compõem o corpo dessa experiência, ora apresentada, está ancorada na Teoria da Intervenção Práxica da Enfermagem em Saúde Coletiva (TIPESC)(8). Essa teoria está estruturada em categorias conceituais (sociedade, homem, processo saúde/doença, saúde coletiva, assistência, enfermagem, trabalho, necessidade, vulnerabilidade e educação) e dimensionais (conjunto de noções utilizadas no processo de seu desenvolvimento). A TIPESC pode ser compreendida como um instrumento teórico-metodológico que possibilita ao trabalhador de enfermagem o exercício de uma crítica preocupada com a mudança do atual modo de organização da sociedade, das políticas de enfrentamento de um fenômeno e das práticas para a intervenção no fenômeno. A aplicação desse instrumento teórico-metodológico se dá em cinco etapas: (1) Captação da realidade objetiva, (2) Interpretação da realidade objetiva, (3) Proposta de Intervenção na realidade objetiva, (4) Intervenção na realidade objetiva, (5) Reinterpretação da realidade objetiva ${ }^{(8)}$.

Na saúde indígena e no contexto da COVID-19, a enfermagem tem função estratégica, exercendo ações de vigilância em saúde, promoção de saúde e cuidado de pessoas e famílias em seus territórios. A descrição dessa experiência seguirá as etapas metodológicas da TIPESC. 


\section{RESULTADOS}

\section{Captação da realidade objetiva}

O DSEl-Manaus abrange uma extensa área territorial $\left(235.405 \mathrm{~km}^{2}\right)$, cobrindo 19 municípios. A população indígena atendida é de 29.506 indivíduos distribuídos em 253 aldeias e em pequenos sítios. O principal meio de transporte de acesso entre a sede dos municípios e a maioria dos Polos-Base é fluvial, cujo tempo de deslocamento pode durar de horas a dias. Alguns rios são navegáveis durante um período do ano (cheia), o que dificulta a oferta de serviço de saúde no outro período do ano (vazante) $)^{(4)}$.

A atenção à saúde das populações indígenas é conduzida na rede hierarquizada e articulada com os pontos da Rede de Atenção à Saúde (RAS), disponível nos âmbitos municipal e estadual. A estrutura dos estabelecimentos do sistema de atenção à saúde indígena para o atendimento em APS é instalada em terras indígenas: Polos-Base e Unidades Básicas de Saúde Indígena. Para o atendimento especializado e hospitalar são utilizados os serviços do SUS destinados à população geral (serviços de referência) ${ }^{(9)}$.

Os Polos-Base abrangem extensões territoriais que agregam um determinado número de aldeias. Sua estrutura é composta por farmácia (medicamentos básicos), sala de observação, sala de curativo e materiais médico-hospitalares, alojamento dos profissionais de saúde, um motor de popa acoplado ao bote de alumínio.

Na sua estrutura de atenção à saúde indígena, o DSEI-Manaus dispõe de uma Casa de Saúde Indígena (CASAI), localizada em Manaus, e três Casas de Apoio, localizadas em três municípios do interior do estado (Autazes, Borba e Manicoré). Esses estabelecimentos constituem a primeira referência para o acesso à atenção especializadaambulatorial ou hospitalar, exceto para os casos de urgência e emergência, para os indígenas procedentes das aldeias. Esses estabelecimentos dispõem de condições funcionais para acolher, alojar, fornecer alimentos e prestar assistência de enfermagem 24 horas por dia. Cabe ainda destacarque são espaços de atendimento e apoio ao indígena e membro de sua família (acompanhante) que nela permaneçam temporariamente para tratamento ou investigação diagnóstica em unidades especializadas de referência ${ }^{(4)}$. Com a finalidade de aproximação cultural, esses estabelecimentos mantêm características ambientais indígenas.

O acesso aos sistemas de comunicação (telefone, radiofonia ou outro meio) é bastante limitado nas aldeias e em outros territórios indígenas. Em algumas aldeias, o telefone pessoal dos profissionais da equipe de saúde e das lideranças indígenas é o único meio de conexão (uso de aplicativo/WhatsApp) com outros serviços fora desses territórios.

Em relação à cultura, a maioria dos grupos étnicos tem contato com populações não indígenas e fala o idioma português fluentemente. No entanto, há grupos menores que mantêm pouco contato com os não indígenas, como, por exemplo, os Mura-Pirahã e os Hexkaryano, que se comunicam quase exclusivamente em língua própria da etnia(4).

Indicadores das condições de saúde referentes a 2019, no âmbito do DSEI-Manaus e com ênfase nas doenças respiratórias e nos fatores de risco para a fase grave da COVID-19 (idade e doenças pré-existentes), mostram que a faixa etária de 60 anos ou mais corresponde a $6,5 \%$ da população (indígena) e prevalências altas de diabetes $(9,6 \%)$, hipertensão $(43,6 \%)$ e de infecções respiratórias $(9,6 \%)^{(4)}$. Os números relativos à COVID-19 mostram que 119 povos indígenas em todo o território brasileiro foram afetados. Até 27/06/2020 havia 5.525 casos confirmados e 134 óbitos. No DSEl-Manaus, até a data citada, eram 212 casos confirmados e sete óbitos ${ }^{(10)}$.

A força de trabalho da enfermagem é outro ponto relevante que compõe a realidade objetiva da saúde indígena. Considerados os nove DSElsque geograficamente pertencem ao Amazonas, a força de trabalho da enfermagem é constituída por 351 enfermeiros e 675 técnicos de enfermagem; no DSEl-Manaus são 56 e 116, respectivamente. Esses trabalhadores têm vínculo trabalhista com Organizações Não Governamentais credenciadas pela Secretaria Especial de Saúde Indígena - SESAI ${ }^{(4)}$. 


\section{Interpretação da realidade objetiva}

O DSEl é responsável pelo planejamento, coordenação, supervisão, monitoramento e avaliação da implementação das ações de saúde, observando os princípios e as diretrizes do SUS e a Política Nacional de Atenção à Saúde dos Povos indígenas. Para adequar essas responsabilidades e atender às necessidades e especificidades locais, perante o agravamento dos problemas sanitários causados pela circulação do Novo Coronavírus e seu alto poder de transmissibilidade entre populações em situação de vulnerabilidade (biológica e social), foram estabelecidas estratégias e ações ${ }^{(4)}$ que vêm sendo desempenhadas pela equipe multidisciplinar de saúde indígena. Neste cenário, destaca-se a atuação dos trabalhadores da enfermagem, por terem papel estratégico tanto nas ações de cuidado, vigilância e promoção da saúde, como nas ações de segurança do paciente e dos trabalhadores de saúde.

\section{Proposta de Intervenção na realidade objetiva}

Com foco na APS e na garantia de integralidade da assistência, pautado nos princípios da diversidade sociocultural e nas particularidades epidemiológicas e logísticas dos povos indígenas dessa área de abrangência, o plano de contingenciamento do DSEI-Manaus foi estruturado em três níveis de resposta ao Novo Coronavírus: alerta (risco de introdução do Novo Coronavírus nos ambientes de atendimento e nas aldeias), perigo iminente (situação de casos confirmados) e emergência em saúde pública (situação epidemiológicas grave) ${ }^{(4)}$. Dada a relevância e o protagonismo da enfermagem na condução das ações no âmbito da APS, optou-se por relatar as ações relativas à vigilância, promoção, cuidado e segurança do paciente e do trabalhador correspondentes ao primeiro nível de resposta no enfrentamento da pandemia, ou seja, o nível nomeado de alerta.

Para a vigilância em saúde foram propostas ações voltadas à qualificação profissional, monitoramento dos casos suspeitos e confirmados, atualização e adequação das orientações, implantação de protocolos, notificação imediata de casos, garantia de direitos e atendimento a demandas clínicas específicas. Para a promoção à saúde foram recomendadas ações de educação em saúde, considerando as especificidades dos territórios e buscando apoio na comunidade indígena e em suas lideranças. Quanto ao cuidado de pessoa e família, foi proposta a continuidade das práticas de atenção à saúde nas unidades ou no domicílio, com especial atenção à identificação de sinais de síndrome gripal e de sua gravidade, orientação para isolamento domiciliar (quando necessário), realização de testagem de sintomáticos. E, em relação à segurança do paciente indígena e do trabalhador,foram propostos momentos de capacitação sobre temas pertinentes diversos (formas de transmissão, medidas de biossegurança individual e coletiva), provisão de estrutura adequada para higienização das mãos dos pacientes, acompanhantes e profissionais de saúde durante a triagem nos atendimentos nos Polos-Base ou em outros espaços.

\section{Intervenção na realidade objetiva}

A equipe de saúde indígena enfrenta diferentes desafios (logístico, geográficos, trabalhistas, pessoais e familiares) para realizar as ações inerentes às suas respectivas habilidades e competências profissionais. A competência cultural é também primordial para que as ações alcancem os resultados esperados e, nessa perspectiva, a enfermagem do DSEI-Manaus tem atuado. (Figura 1).

No Quadro 1 são apresentadas as ações do primeiro nível de resposta (alerta) relativas às quatro dimensões selecionadas que vêm sendo realizadas pela equipe de enfermagem no enfrentamento da pandemia do Novo Coronavírus entre populações indígenas do DSEI-Manaus. 


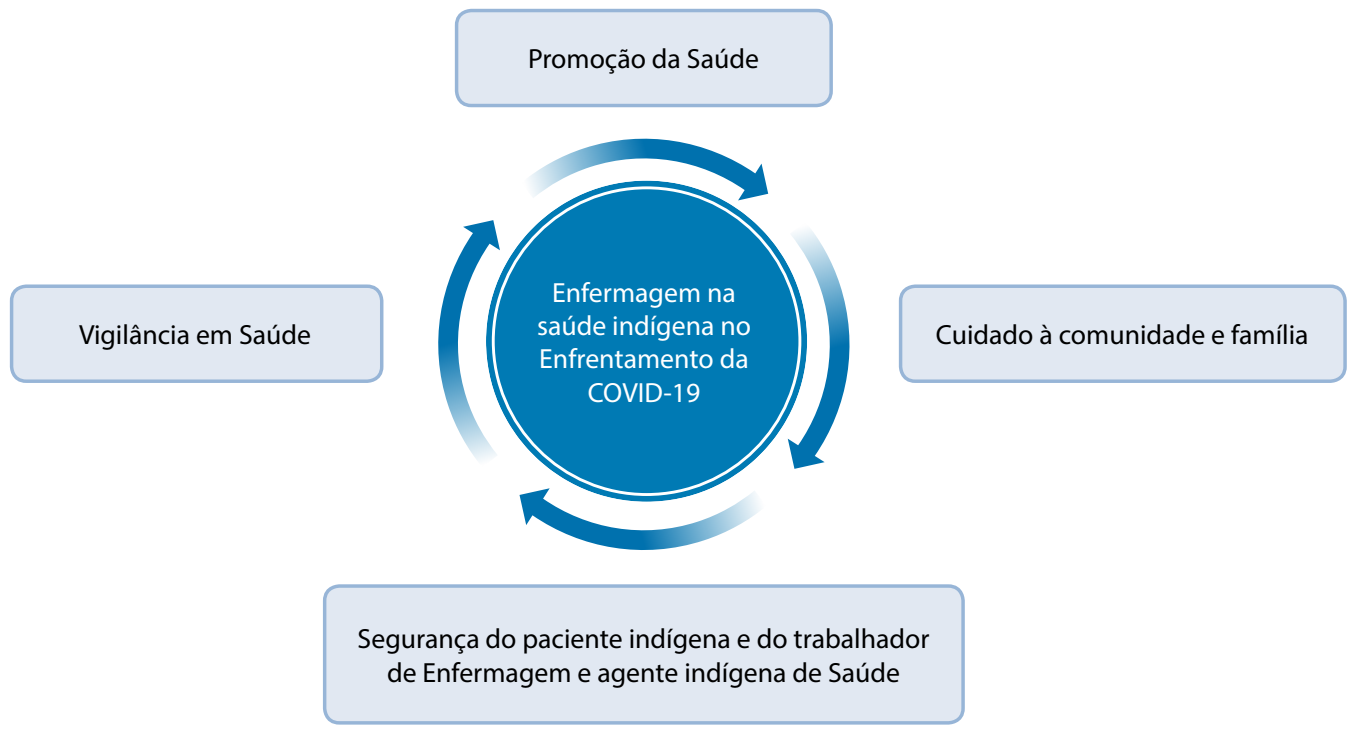

Figura 1 - Diagrama de atividades conduzidas pela enfermagem em território indígena, Distrito Sanitário Especial Indígena - Manaus

Quadro 1- Ações desenvolvidas pela Enfermagem em resposta às demandas geradas pela circulação do Novo Coronavírus no âmbito do Distrito Sanitário Especial Indígena - Manaus

\begin{tabular}{|c|c|c|c|}
\hline Vigilância à Saúde & Promoção de Saúde & $\begin{array}{l}\text { Cuidado de pessoas/ } \\
\text { família e comunidade }\end{array}$ & $\begin{array}{l}\text { Segurança de Indígenas e } \\
\text { trabalhadores de Saúde }\end{array}$ \\
\hline $\begin{array}{l}\text { Conhecimento da situação } \\
\text { de saúde das famílias e } \\
\text { comunidades indígenas } \\
\text { (atualização de informações } \\
\text { de saúde); } \\
\text { Identificação de casos } \\
\text { suspeitos e orientação } \\
\text { quanto ao isolamento } \\
\text { domiciliar; } \\
\text { Atualização de definições e } \\
\text { critérios de notificação; } \\
\text { Manutenção do padrão de } \\
\text { notificaçáo rápida dos casos } \\
\text { suspeitos e confirmados, } \\
\text { para fins de atualização do } \\
\text { boletim epidemiológico; } \\
\text { Planejamento e execução } \\
\text { de treinamento com foco na } \\
\text { vigilância em saúde para os } \\
\text { profissionais de nível técnico; } \\
\text { Investigação dos casos } \\
\text { confirmados e rastreamento } \\
\text { dos contatos de casos } \\
\text { suspeitos; } \\
\text { Testagem dos casos } \\
\text { suspeitos; } \\
\text { Capacitação de lideranças } \\
\text { indígenas e dos conselheiros } \\
\text { distritais com foco na adoção } \\
\text { de medidas de prevenção da } \\
\text { COVID-19. }\end{array}$ & $\begin{array}{l}\text { Envolvimento das } \\
\text { comunidades, por meio de } \\
\text { suas lideranças, nas estratégias } \\
\text { promotoras de saúde; } \\
\text { Divulgação sistemática de } \\
\text { informações sobre medidas de } \\
\text { proteção individual e coletiva, } \\
\text { contra o Coronavírus } \\
\text { Intensificação da comunicação } \\
\text { nas aldeias, utilizando e } \\
\text { otimizando os recursos } \\
\text { existentes na comunidade } \\
\text { (rádios, radiofonia, aplicativos, } \\
\text { grupos de WhatApp). } \\
\text { Produção coletiva } \\
\text { (profissionais e comunidade) } \\
\text { de estratégias viáveis para } \\
\text { garantir o distanciamento } \\
\text { social nas aldeias; } \\
\text { Orientação e treinamento } \\
\text { para a população sobre uso de } \\
\text { máscaras de tecido e outras } \\
\text { medidas de proteção (higiene } \\
\text { pessoal, lavagem das mãos, } \\
\text { etiqueta respiratória); } \\
\text { Tomada de decisão coletiva, } \\
\text { envolvendo lideranças } \\
\text { indígenas, para criação de } \\
\text { espaços físicos destinados ao } \\
\text { isolamento de casos positivos } \\
\text { e suspeitos ou para indivíduos } \\
\text { de maior vulnerabilidade para } \\
\text { Covid-19. }\end{array}$ & $\begin{array}{l}\text { Envolvimento de lideranças } \\
\text { indígenas no planejamento } \\
\text { de atividades; } \\
\text { Atendimento da demanda } \\
\text { livre ou por busca ativa no } \\
\text { âmbito dos Polos-Base com } \\
\text { suspeita ou confirmação de } \\
\text { Covid-19; } \\
\text { Provimento de material } \\
\text { para o trabalho da equipe } \\
\text { de enfermagem (oxímetros, } \\
\text { fonte de O², equipamentos } \\
\text { de proteção individual - EPIs, } \\
\text { entre outros itens); } \\
\text { Realização de testagem para } \\
\text { Covid-19; } \\
\text { Realização de visitas } \\
\text { domiciliares, priorizando } \\
\text { idosos ou outros grupos } \\
\text { com comorbidades, } \\
\text { imunodeprimidos, gestantes, } \\
\text { puérperas/recém-nascidos; } \\
\text { Manutenção do diálogo } \\
\text { intercultural com sujeitos } \\
\text { e comunidades indígenas, } \\
\text { respeitando e valorizando os } \\
\text { conhecimentos tradicionais. }\end{array}$ & $\begin{array}{l}\text { Previsão e provisão de EPIs; } \\
\text { Utilização de EPIs nos } \\
\text { ambientes de trabalho e de } \\
\text { convívio social/coletivo; } \\
\text { Capacitação dos profissionais } \\
\text { de enfermagem e agentes } \\
\text { indígenas de saúde, com foco } \\
\text { no uso correto dos EPIs; } \\
\text { Capacitação para os } \\
\text { trabalhadores técnicos } \\
\text { (técnicos de enfermagem e } \\
\text { agentes indígenas de saúde), } \\
\text { com foco no reconhecimento } \\
\text { das formas de transmissão } \\
\text { da doença e das medidas } \\
\text { individuais e coletivas de } \\
\text { proteção; } \\
\text { Realização de testagem para } \\
\text { COVID-19 dos trabalhadores } \\
\text { de enfermagem; } \\
\text { Manutenção de ambientes } \\
\text { (Polo-Base) seguros para os } \\
\text { profissionais e população } \\
\text { indígena. }\end{array}$ \\
\hline
\end{tabular}




\section{Reinterpretação da realidade objetiva}

As estratégias e ações planejadas e implementadas são avaliadas segundo critérios internosdo DSEI-Manaus. No que tange à transmissão e adoecimento pelo novo Coronavírus, a avaliação se assenta em dados epidemiológicos (número de casos suspeitos e confirmados e de óbitos). Reconhecidas a vulnerabilidade da população indígena e suas dificuldades de acesso aos serviços de saúde, os profissionais da saúde, especificamente os da enfermagem, seguem no processo de aplicação do plano de intervenção, a fim assegurar a oferta de assistência focada na integralidade do cuidado que alcance a proteção contra a transmissão do vírus (COVID-19) e a promoção da saúde nos territórios indígenas em que atuam.

Constata-se que tem havido mudanças nos costumes e práticas tradicionais de cura e cuidadoentre os grupos étnicos, nas aldeias. Nesse sentido, associado à prática do isolamento social, muitos preferem utilizar seus remédios caseiros, porque acreditam na prevenção da COVID-19 e na sua cura. Assim, os profissionais de enfermagem, e em especial os agentes indígenas de saúde, atuam também na negociação e conciliação com os grupos étnicos, detentores de "saber tradicional", para implementar o cuidado integral preconizado oficialmente, o "saber científico". Essa realidade objetiva das comunidades indígenas vem permitindo ao trabalhador da enfermagem pensar o cuidado culturalmente diferenciado. Dentre as prioridades nesse contexto, a saúde mental da população indígena e dos profissionais da equipe de saúde requer maior atenção. As situações concretas geradoras de sofrimento mental estão relacionadas ao manejo de pacientes graves e de corpos (retorno do corpo às aldeias, rituais de sepultamento) e ao luto nos diferentes grupos étnicos e, também, à exclusão de determinados grupos etários quando adoecidos, em geral idosos e crianças.

\section{DISCUSSÃO}

No campo da realidade objetiva, a precária estrutura dos serviços de saúde na região, configurada pela ausência de hospitais regionais de referência, de infraestrutura adequada para atendimento aos pacientes graves de COVID-19 e pela escassez de profissionais qualificados para o manejo clínico desses casos, constitui-se em um dos mais complexos desafios na atenção à saúde dos povos indígenas no Amazonas. Assim, o enfrentamento à COVID-19 nos territórios indígenas do DSEI-Manaus tem sido dependente quase que exclusivamente da APS, o que exige das instâncias de gestão (SESAI e DSEI) estratégias bem definidas na previsão e provimento de infraestrutura, logística, equipamentos, insumos, recursos humanos e, principalmente,na qualificação dos trabalhadores de enfermagem, visto que a enfermagem está na linha de frente em todas as dimensões da atenção em saúde destacadas e tratadas no presente relato (vigilância, promoção da saúde, cuidado integral e segurança de povos indígenas e trabalhadores de saúde). Por isso, a implementação do primeiro nível de resposta no enfrentamento da pandemia nos territórios indígenas tem sido essencial para conter a circulação do Novo Coronavírus.

Para realizar a intervenção na realidade objetiva, a equipe de saúde também enfrenta desafios que impactam no controle do avanço da pandemia nos territórios indígenas. Dentre os quais se destacam as especificidades socioculturais e limitações de acesso aos serviços de saúde em quase todos os grupos étnicos que vivem na região. Outra fragilidade vivenciada pela equipe é o controle das morbidades pré-existentes, como as doenças infecciosas e crônicas não transmissíveis que, por sua vez, tornam essa população ainda mais vulnerável à COVID-19. Além disso, o acesso das equipes de saúde às comunidades indígenas tem se configurado como um desafio, devido às barreiras sanitárias dos munícipios e à resistência de povos indígenas nos seus territórios frente à entrada de pessoas vindas de áreas onde a transmissão comunitária foi instalada. Paradoxalmente, essas barreiras têm impedido ou retardado, também, a entrada de profissionais das equipes de saúde indígena em seus territórios sanitários.

Contudo, a articulação dos agentes indígenas de saúde com as lideranças indígenas, para o monitoramento de entrada nos territórios indígenas de pessoas com ou sem sintomas respiratórios, as restrições relativas à 
saída dos moradores e as estratégias de isolamento dos casos suspeitos ou confirmados, especialmente nas aldeias com grande número de habitantes, têm se mostrado eficazes na contenção do número de casos.

As medidas individuais e coletivas de prevenção, como confecção e uso da máscara facial de pano, distanciamento social, uso individual de utensílios e objetos pessoais, bem como a adequada higienização das mãos com água e sabãosão pontos debatidos sistematicamente nas aldeias, inclusive naquelas mais distantes das áreas urbanizadas. Entre os povos de recente contato e isolados, as medidas são mais voltadas ao controle de acesso ao território e medidas de isolamento (quarentena dos profissionais com ou sem sintomas respiratórios, ao entrarem no território) e teste diagnóstico. Também a identificação de casos sintomáticos para síndrome gripal têm possibilitado à equipe de saúde o acompanhamento e a intervenção (encaminhamento) precocemente. Os casos graves que necessitam de tratamento hospitalar, principalmente aqueles que precisam de suporte ventilatório, são removidos para capital (Manaus), única cidade do estado onde há serviço de terapia intensiva.

Cabe destacar que a sistematização do cuidar em saúde indígena, no que se refere ao fazer dos trabalhadores da enfermagem (identificação de problemas, o processo de julgamento e os resultados de enfermagem), foi estruturada considerando a realidade cultural dos grupos étnicos envolvidos. Embora todos os cuidados estejam pautados nas melhores evidências científicas, a enfermagem precisa sempre estabelecer uma relação dialógica de confiança e afetiva com os indígenas, para favorecer o alcance dos resultados e a compreensão da realidade que pautem práticas mais avançadas no sentido de obtenção de proteção da saúde e controle de doenças, incluindo a COVID-19, entre essas populações.

A experiência da enfermagem em saúde indígena tem indicado que é relevante manter a atenção sobre a realidade (necessidade) desses profissionais. Suas vivências têm sido fundamentais na gestão (planejamento, monitoramento, avaliação) e na implementação das ações (cuidado de pessoas, família e comunidade, incluindo as ações de vigilância em saúde), contribuindo, desse modo, para o planejamento de outras estratégias de impacto positivo nas condições de saúde dos indígenas. Entretanto, esses profissionais não têm vínculo trabalhista estatal (seus vínculos são com organizações sociais).

Em todas as situações, a articulação da equipe,e em especial dos agentes indígenas de saúde,com as lideranças indígenas tem sido essencial para o fortalecimento das ações de vigilância e promoção da saúde, especialmente as que são exercidas pelos profissionais da enfermagem, os quais representam o maior contingente de trabalhadores atuando na linha de frente no combate à pandemia.

\section{CONCLUSÃO}

Essa experiência permitiu ampliar a percepção quanto à importância do uso de um modelo teórico-metodológico para planejar, executar e avaliar ações de enfermagem. Permitiu também notar que[,]mesmo em um cenário geográfico marcado por adversidades, com previsões epidemiológicas desfavoráveis, principalmente para os territórios indígenas, e sendo essas populações pertencentes aos grupos vulneráveis à forma grave da COVID-19, os resultados do trabalho da enfermagem, nos campos da vigilância e promoção da saúde, têm contribuído para minimizar ou evitar o avanço da doença e impactado na redução gradativa da sua letalidade, também entre os indígenas.

Nessa perspectiva, espera-se que a experiência descrita possa subsidiar profissionais de enfermagem que atuam na saúde indígena, provocando reflexão sobre a sistematização das ações de enfermagem acerca da vigilância em saúde, promoção da saúde em território indígena, cuidado integral à família e comunidade, bem como a segurança dos usuários e trabalhadores, no âmbito da área de abrangência dos Distritos Sanitários Especiais Indígenas.

\section{REFERÊNCIAS}

1. Brasil. Ministério da Saúde. Boletim epidemiológico COVID-19, n..3 [ Internet]. 2020. [cited 2020 17]. Available from: https:// portalarquivos2.saude.gov.br/images/pdf/2020/fevereiro/21/2020-02-21-Boletim-Epidemiologico03.pdf 
2. Fundação de Vigilância do Amazonas. BoletimCovid, n.10 [Internet]. 2020. [cited 2020 21]. Available from: http:// transparencia.fvs.am.gov.br/media/publicacao/boletim_covid_10.pdf

3. Codeço CT, Villela D, Coelho F, Bastos LS, Gomes MFC, Cruz OG, et al. Estimativa de risco de espalhamento da COVID-19 no Brasil e o impacto no sistema de saúde e população por microrregião. Rio de Janeiro: Fiocruz/PROCC, 2020. 3v. [cited 2020 21].Availablefrom:https://www.arca.fiocruz.br/handle/icict/40509

4. Distrito Sanitário Especial Indígena de Manaus. Plano de contingência para infecção humana pelo novo coronavírus (covid-19) em povos indígenas do DSEl Manaus, Manaus, 2020, 50 p.

5. Instituto Brasileiro de Geografia e Estatística. Indígenas. 2020: Brasil. [cited 2020 jun 18]. Available from: https://indigenas. ibge.gov.br/

6. Rocha ESC, Gonçalves MJF, CRUZ, MJF, Lacerda RAB.Perfil de enfermeiros (as) que atuam na saúde indígena e não indígena/Profile of nurses whowork in indigenousand non-indigenoushealthcare. CiencCuidSaude , 2018; 17(4): 45195. DOI: https://doi.org/10.4025/cienccuidsaude.v17i4.45195

7. Conselho Federal de Enfermagem. Enfermagem. Nota de Esclarecimento sobre o Coronavírus (COVID-19).[cited 2020 jun 11]. Available from: http://www.cofen.gov.br/cofen-publica-nota-de-esclarecimento-sobre-o-coronavirus-covid-19_77835.html.

8. Egry EY, Fonseca RMGS, Oliveira MAC, Bertolozzi MR. Enfermagem em Saúde Coletiva: reinterpretação da realidade objetiva por meio da ação praxiológica. RevBrasEnferm [Internet]. 2018;71(supl1):758-63. doi: http://dx.doi. org/10.1590/0034-7167-2017-0677.

9. Secretaria Especial de Saúde Indígena. Departamento de Atenção à Saúde Indígena. Nota Técnica no 17 de 29 de agosto de 2018. Documento orientador da organização dos processos de trabalho da atenção primária à saúde nos Distritos Sanitários Especiais Indígena, Brasília, 2018.

10. Secretaria Especial de Saúde Indígena. Departamento de Atenção à Saúde Indígena. Boletim Epidemiológico da SESAI, 2020 [cited 2020 jun 29] Availablefrom:https://saudeindigena.saude.gov.br/. 